\title{
Analysis of the Isothermal Forced Flow Chemical Vapour Infiltration Process. Part I: Theoretical Aspects
}

\author{
Y. G. Roman, ${ }^{a}$ J. F. A. K. Kotte ${ }^{a} \&$ M. H. J. M. de Croon ${ }^{b}$ \\ "TNO, Centre for Technical Ceramics, PO Box 595, 5600 AN Eindhoven, The Netherlands \\ ${ }^{b}$ Eindhoven University of Technology, Department of Chemical Engineering, PO Box 513, 5600 MB Eindhoven, \\ The Netherlands
}

(Received 24 June 1994; revised version received 2 March 1995; accepted 10 March 1995)

\begin{abstract}
In this paper the densification of a cylindrical pore due to a first order heterogeneous deposition reaction on the wall is described. The basic understanding of these mechanisms can aid in the prediction of the infltration time and composite porosity after densification. The results of the modelling are compared with experimental results which are reported in an additional paper, i.e. Part 2 of this study. The main characteristic parameter that determines the infiltration behaviour is the gas velocity.
\end{abstract}

\section{Scope}

The drive to gain 'tough' ceramic material led to the development of the so-called thermal gradient FCVI (TGFCVI) technique some ten years ago. ${ }^{1-4}$ With this method, thick walled ceramic component structures can be made. A drawback however, is that for each different sample geometry, a new fixture needs to be developed through which a thermal gradient can be applied across the fibrous preform.

The isothermal FCVI (IFCVI) method does not have this problem, so the fixture can have a much simpler design. For thin walled structures like hot gas filters, heat shields, burner tubes, seal rings and heat exchanger panels, this isothermal method can thus provide a great improvement towards industrial application of the FCVI technique. Optimisation of IFCVI process conditions however is of great importance to be able to homogeneously fill the porous substrate, especially in the direction parallel to the gas flow.

The modelling of both ICVI and FCVI processes is a topic of much effort and a first attempt to optimise conditions rapidly. However, the experimental validation of these models (especially FCVI) is still very limited and a basic insight into the process mechanisms is often arduous. As far as is known, there are no publications on the effect of the different process variables of the FCVI process on the infiltration efficiency in terms of composite density and infiltration time. This study is an attempt to fill this gap and reach a better understanding in the IFCVI process using on one hand a very simple model (viz. in Part 1) and on the other hand, experimental validation (Part 2). ${ }^{5}$

\section{Introduction}

When reactive precursor gas is forced through a heated porous fibrous preform, a chcmical reaction takes place on the fibre surface and a solid matrix material is deposited onto the fibres: the forced flow chemical vapour infiltration (FCVI) process.

In this study, silicon carbide ( $\mathrm{SiC}$ ) is deposited onto the fibres as a result of a heterogeneous decomposition of methyl-trichlorosilane (MTS) in hydrogen:

$$
\mathrm{SiCH}_{3} \mathrm{Cl}_{3(\mathrm{~g})}+\mathrm{H}_{2(\mathrm{~g})} \rightarrow \mathrm{SiC}_{(\mathrm{s})}+3 \mathrm{HCl}_{(\mathrm{g})}+\mathrm{H}_{2(\mathrm{~g})}
$$

As the material is growing onto the fibres, the density of the preform increases and the pores get smaller. The preform microstructure is thus changing with time.

In principle, the preform can be visualised simply as a cylindrical plate in which cylinder holes (pores) are present. Through these pores the gas flows, due to the pressure gradient that is present across the plate. The diffusion duc to concentration gradients along the pore axis can be neglected, because it is much smaller than the mass transport of the species due to the pressure driven convection.

The FCVI process is dependent on two kinds of parameters, i.e. the process conditions such as:

- Temperature of the preform

- Total pressure

- Gas velocity 
- Precursor concentration

and the microstructural properties of the starting preform such as:

- Preform permeability

- Preform porosity

- Average pore size and pore size distribution

- Preform thickness

- Pore tortuosity

- Specific surface area available for deposition.

For a complete understanding of the FCVI process it is necessary to know the effect on the infiltration process when one or more of these parameters are varied.

The purpose of this manuscript is to gain more understanding of the infiltration behaviour and this paper describes the effect of the different process conditions and microstructural properties of the preform under isothermal conditions. Although the model presented is a simplification of reality, it can provide insight into the basic principles of the FCVI process.

\section{One-dimensional analysis of the isothermal CVI process}

The three dimensional raw fibrous preforms used in Part 2 of this study, contain pores of three size ranges and contain an average total porosity of 50 vol $\%$. The smallest pores are smaller than 0.01 $\mu \mathrm{m}$, bigger pores between $2-30 \mu \mathrm{m}$ represent the pores within the bundles and large pores with a diameter between $100-1000 \mu \mathrm{m}$ are the voids between two crossing tows and the pores between the tows.

When it is assumed that the relative contribution in void space of the two smallest pores is negligible, then the porosity will depend on the large pores only. As such, the infiltration time and densification of the preform is dependent on these large pores, since they determine the preform permeability and pressure drop and thus the moment that the process is terminated, i.e. when the pore entrances of these big pores are (almost) closed due to matrix deposition.

For the description of the CVI process. the preform can then be represented simply as a plate with continuous monosized pores of $500 \mu \mathrm{m}$ in diameter under isothermal conditions. The pores are straight and open on both sides. Such a model preform is visualised in Fig. 1.

Distinction should be made in the way the gas species are transported into the pore cavity using one of the different CVI methods. During isothermal isobaric CVI (ICVI) processing, the gas flows around the model preform and the gas species are transported into the pores by diffusion.

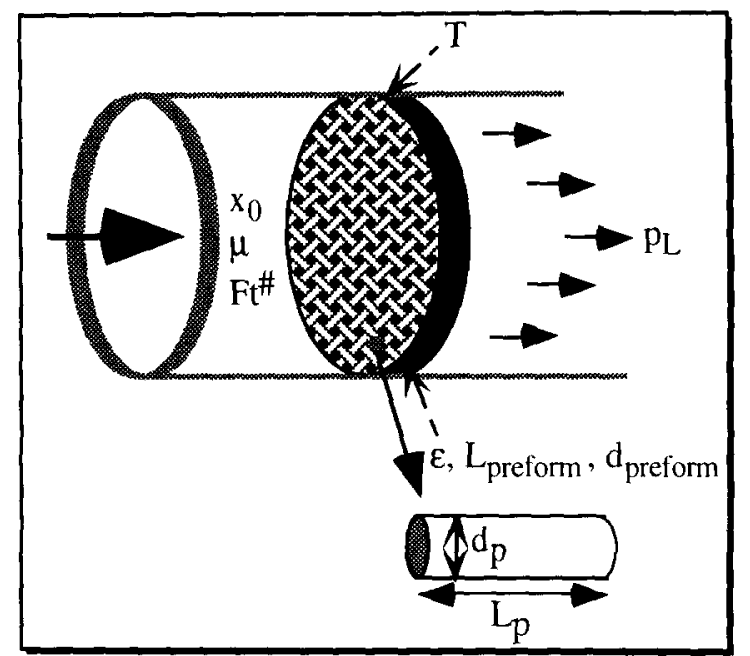

Fig. 1. The cross-section of the reactor tube with a porous model preform consisting of $n$ monosized cylindrical pores. Known initial adjustable process and microstructural parameters are indicated.

On the other hand, in the case of isothermal forced flow CVI (IFCVI), the gas mixture enters the preform from one side and flows in axial direction due to forced convection.

For both ICVI and IFCVI densification, silicon carbide $(\mathrm{SiC})$ is deposited onto the pore wall. The deposition rate of $\mathrm{SiC}$ is controlled by either the chemical reaction rate or one of the two mass transport mechanisms.

Two dimensionless numbers, i.e. the mass Peclet number $(P e)$ and the Damköhler $(D a)$ number ${ }^{1.2}$ describe the competition between the chemical reaction rate at the fibre surface and the mass transport rate of the precursor species into the pore.

The Peclet number describes the ratio of mass transport due to convection into the pore relative to diffusive transport. The Damköhler number is the ratio of the chemical deposition rate of the matrix material relative to the rate of mass transport into the pore. For a porous preform consisting of cylinder pores in which deposition takes place, the $P e$ number for a cylindrical pore is defined as:

$$
P e=\frac{\text { convective mass transport }}{\text { diffusive mass transport }}=\frac{u_{\mathrm{p}} d_{\mathrm{p}}}{D_{\mathrm{eff}}}
$$

$D_{\text {eff }}$ is the effective gas diffusion coefficient $\left(\mathrm{m}^{2} \cdot \mathrm{s}^{-1}\right)$, $d_{\mathrm{p}}$ is the pore diameter $(\mathrm{m})$ at the gas entrance side and $u_{p}$ is the average superficial gas velocity $\left(\mathrm{m} . \mathrm{s}^{-1}\right)$ for laminar flow within a horizontal cylindrical straight pore and will be described later on.

At low Peclet numbers, (i.e. if $u_{\mathrm{p}} d_{\mathrm{p}}<<D_{\mathrm{eff}}$ ), diffusion is the mass transport mechanism whereas at large $P e$ numbers convection is dominant.

The Damköhler number is dependent on the mass transport mechanism and therefore distinction is made between the first and second 
Damköhler number Da I and Da II, respectively). For a first order reaction (in MTS) on the pore wall, these are defined as:

$D a \mathrm{I}=\frac{\text { chemical reaction rate }}{\text { convective mass transport rate }}=\frac{4 k_{\mathrm{s}}}{u_{\mathrm{p}}}$

$D a$ II $=\frac{\text { chemical reaction rate }}{\text { diffusive mass transport rate }}=\frac{4 k_{\mathrm{s}} d_{\mathrm{p}}}{D_{\mathrm{eff}}}$

Here, $k_{\mathrm{s}}$ is the first order reaction rate coefficient based on surface area $\left(\mathrm{m} \cdot \mathrm{s}^{-1}\right)$.

When the Damköhler numbers are small (i.e. if numerator $<<$ denominator), the chemical reaction is rate limiting whereas at large values the rate of mass transport is controlling the $\mathrm{SiC}$ growth.

Four idealised, extreme cases can be given:

(1) The growth rate is mass transport limited and mass transport is dominated by:

(a) gas phase diffusion; Pe small, Da II high;

(b) forced convection; Pe high, Da I high.

(2) The chemical reaction rate is limiting the growth and mass transport is due to:

(a) gas phase diffusion; Pe small, $D a$ II small;

(b) forced convection; Pe high, Da I small.

Cases 1(a) and 2(a) are conditions during the ICVI process (isothermal and isobaric CVI, low $\mathrm{Pe}$ ) and 1 (b) and 2(b) (high $\mathrm{Pe}$ ) are common for FCVI. In the former cases, the gas flows around the substrate and the concentration gradient is the driving force for the diffusion of the precursor gas into the pores. In the latter cases, a pressure gradient across the substrate forces the gas to flow through the pores.

For both situations an equation can be found for the MTS concentration profile (or effectiveness factor $\zeta$ ) within a cylindrical pore (open on both sides) where gas molecules enter the pore from one side. For ICVI (cases 1(a) and 2(a)), diffusion and reaction the effectiveness factor is given in literature. $^{3-5}$

For FCVI (cases 1(b) and 2(b)), forced convection and chemical reaction in a straight pore, the effectiveness factor is defined as (Appendix A):

$$
\frac{C_{\mathrm{z}}}{C_{0}}=\exp \left(\frac{-4 k_{\mathrm{s}} z}{d_{\mathrm{p}} u_{\mathrm{p}}}\right)=\exp \left(\frac{-D a I z}{d_{\mathrm{p}}}\right)
$$

The decrease of the layer thickness or precursor conversion (depletion) along the pore axis from opening $(z=0)$ to end $(z=L)$ can be represented by $\Delta$, defined as: ${ }^{4}$

$$
\Delta=\frac{G_{0}-G_{\mathrm{L}}}{G_{0}}=1-\frac{C_{\mathrm{L}}}{C_{0}}
$$

with $G$ being the first order $\mathrm{SiC}$ growth rate (mol. $\mathrm{m}^{-2} \cdot \mathrm{s}^{-1}$ ) and is:

$$
G_{\mathrm{SiC}}=k_{\mathrm{s}} C_{\mathrm{MTS}, \mathrm{z}}
$$

For FCVI, the deposition layer gradient or depletion can be expressed as:

$$
\Delta=1-\exp \left(\frac{-4 k_{\mathrm{s}} L}{d_{\mathrm{p}} u_{\mathrm{p}}}\right)=1-\exp \left(\begin{array}{c}
-D a \mathrm{I} L \\
d_{\mathrm{p}}^{-}
\end{array}\right)
$$

For a uniform deposited layer, $\Delta$ equals zero. As the precursor conversion is low, the SiC layer uniformity and composite density will thus be large.

For both ICVI and FCVI this means that respectively $D a$ II and $D a$ I should be small. Infiltration should therefore be performed in the reaction rate limited growth regime to enhance uniform deposition.

Furthermore, for ICVI a small $\Delta$ implies a large diffusion coefficient and pore diameter and small pore length. The modelling of the ICVI process is performed by many ${ }^{4-8}$ and is no topic of this study.

\section{The Infiltration Behaviour Versus IFCVI Process Conditions. Introduction}

In this section, some general relationships are derived that are applicable for the description of the IFCVI process. The FCVI process has been modelled by many, using complex and powerful computer models. ${ }^{9-12}$ However, basic insight in the controlling phenomena of the FCVI process is often lost and the model is a black box where a certain input will yield an output that is not well understood. Furthermore, these models are often based upon process variables that are impossible to alter in real experiments. The operator of the IFCVI process is only able to vary four input process conditions, i.e. preform temperature: $T$, total gas flow rate at STP: $F t^{\#}$, total pressure at the gas exit side of the preform: $p_{\mathrm{L}}$ and the reactant mole fraction in the gas mixture at the gas inlet side: $X_{0}$. Furthermore, the shut off point of the process can be adjusted. The latter is a certain pressure difference between the pressure at the gas inlct side at $t=0$ and the pressure at the same location after a densification time period.

In the next section, some simple analytical tools are presented ${ }^{16}$ that can aid in the prediction of trends in matrix growth rate at the gas inlet and outlet position of the preform. First (Section 4.1-4.3), the effects of the four input process conditions are examined. Subsequently, a correlation of the microstructural parameters of the raw (uninfiltrated) preform on the infiltration behaviour is presented in Section 5.

\subsection{General description of the growth rate during FCVI in a cylindrical model pore}

The growth rate $G_{z}\left(\mathrm{~mol} \cdot \mathrm{m}^{-2} \cdot \mathrm{s}^{-1}\right)$ of the solid matrix material $\mathrm{SiC}$ in axial direction $z$ in the pore is given by: 


$$
G_{\mathrm{z}}=C_{\mathrm{z}} k_{\mathrm{s}}=C_{0} k_{\mathrm{s}} \exp \left(\frac{-4 k_{\mathrm{s}} z}{d_{\mathrm{p}} u_{\mathrm{p}}}\right)
$$

As infiltration proceeds, the pore mouth - of diameter $d_{\mathrm{p}}(m)($ at $\mathrm{z}=0)$ - will become smaller due to deposition, hence:

$$
d_{\mathrm{pt}}=d_{\mathrm{p} 0}-\frac{2 t_{\mathrm{i}} G_{0} m_{\mathrm{SiC}}}{p_{\mathrm{SiC}}}
$$

Here, $m_{\mathrm{SiC}}$ is the molar mass of $\mathrm{SiC}=0.040$ kg.mol ${ }^{-1}$ and $\rho_{\mathrm{SiC}}=3.2 \times 10^{3} \mathrm{~kg} \cdot \mathrm{m}^{-3}, t_{\mathrm{i}}$ is the infiltration time (s).

\subsection{Kinetically controlled matrix growth}

At very largc gas velocities ( $u_{\mathrm{p}}>$ infinity), eqn 8 reduces to $G_{\mathrm{z}}=C_{0} k_{\mathrm{s}}=G_{0}$, indicative of kinetically controlled growth. Within this growth regime, $\Delta$ (eqn 7 ) equals 0.

$$
G_{\mathrm{z}}=k_{\mathrm{s}} C_{\mathrm{z}}=k_{0} \frac{X_{0} p_{0}}{\operatorname{Rg} T} \quad \exp \left(\frac{-E a}{\operatorname{Rg} T}\right)
$$

with $R g\left(\mathrm{~J} \cdot \mathrm{mole}^{-1} \cdot \mathrm{K}^{-1}\right)$ as the gas constant and $E a$ (J.mole $\left.{ }^{-1}\right)$ the activation energy of the heterogeneous reaction. Clearly, neither the total gas flow rate does not affect the growth rate within the pore. Increase of total inlet pressure $p_{0}$, temperature $T$ or molar fraction of the precursor $X_{0}$, will increase the growth of $\mathrm{SiC}$.

Theoretically, compared with kinetically controlled growth during ICVI, FCVI will not allow complete densification of the preform due to the imposed pressure gradient that yields a deposition gradient.

\subsection{Forced convection controlled matrix growth}

The effect of the process conditions in case of mass transport limited $\mathrm{SiC}$ growth, is not as straightforward as for kinetically controlled growth. The gas velocity is the prime parameter that determines the growth rate of $\mathrm{SiC}$ along the pore axis.

\section{Gas velocity}

Clearly, the gas velocity is of prime importance during convective mass transport. The gas velocity determines the mass transport rate of the MTS and depletion along the pore axis. This pressure driven velocity of the precursor species is represented by the gas velocity $u_{\mathrm{p}}$ of the gas through a pore $p$ of the preform.

As a starting point, the superficial gas velocity $u$ $\left(\mathrm{m} . \mathrm{s}^{-1}\right)$ for a hollow cylinder tube of constant diameter, based on the mass flux of the species is defined as:

$$
u=\frac{F t}{A}=\frac{F t^{\#} T p^{0}}{T^{0} \bar{p} A}
$$

Here, $F t^{\#}$ is the total gas flow rate at STP conditions $\left(\mathrm{m}^{3} \cdot \mathrm{s}^{-1}\right), A$ is the cross flow area of the cylin- drical tube and is thus $0.25 \pi d^{2}\left(\mathrm{~m}^{2}\right), d$ is the tube diameter $(\mathrm{m}), T^{0}$ and $p^{0}$ are the standard temperature $(273 \mathrm{~K})$ and standard pressure $\left(10^{5} \mathrm{~Pa}\right)$ respectively, $\bar{p}$ is the arithmetic mean pressure, averaged across the preform width $(\mathrm{Pa})$. This description of the gas velocity in the reactor tube or pore is linearly dependent on the gas inflow rate $F t$.

As a first approximation it is assumed that the pressure within the pore decreases linearly from gas inlet to outlet of the preform and the gas velocity through the pore is the gas velocity based upon the pore mouth diameter. In other words, these assumptions imply that the pore becomes smaller, but remains of uniform diameter.

The average total pressure in the pore $\bar{p}$ is:

$$
\bar{p}=\frac{p_{0}+p_{\mathrm{L}}}{2}
$$

where $p_{0}$ is the pressure at the gas inlet side of the preform and $p_{\mathrm{L}}$ the pressure at the gas outlet side $(\mathrm{Pa})$.

The average ${ }^{\dagger}$ gas velocity $u_{\mathrm{p}}\left(\mathrm{m} \cdot \mathrm{s}^{-1}\right)$, according to the Hagen-Poiseuille relationship ${ }^{1}$ for a hollow cylindrical straight pore $p$ where laminar flow is present and the gas density is constant (incompressible flow) is given by:

$$
u_{\mathrm{p}}=\frac{d_{\mathrm{p}}^{2} \Delta p}{32 \mu \mathrm{L}}
$$

Here $L(\mathrm{~m})$ is the pore length and equals (in this case, Fig. 1) the preform thickness, $\mu(\mathrm{Pa} . \mathrm{s})$ is the dynamic viscosity of the gas mixture flowing through the pore, and $\Delta p(\mathrm{~Pa})$ is the pressure drop across the pore or preform:

$$
\Delta p=p_{0}-p_{\mathrm{L}}
$$

The pore gas velocity, (i.e. the average gas velocity through each of the total of $n$ single monosized straight pores) is linked with the superficial gas vclocity through a hollow IFCVI reactor tube $u$, through the preform total porosity $\epsilon$ :

$$
u_{\mathrm{p}}=\frac{u}{\epsilon}
$$

The preform porosity $\epsilon$ (volume fraction) is derived from the total pore volume equation. The total pore volume of a preform equals $n \times V_{\mathrm{p}}$ or $\epsilon \times V_{\text {preform }}$ and hence the (constant) number of pores $n$, with constant diameter $d_{\mathrm{p}}$ is:

$$
n=\frac{\epsilon d_{\text {preform }}^{2} L_{\text {preform }}}{d_{\mathrm{p}}^{2} L_{\mathrm{p}}}
$$

where $L_{\text {preform }}$ can be considered to equal $L_{\mathrm{p}}$, when the tortuosity of the pore equals one. $V_{\mathrm{p}}$ is the

${ }^{\dagger}$ The average velocity of the parabolic velocity profile in the hollow tube or pore. 


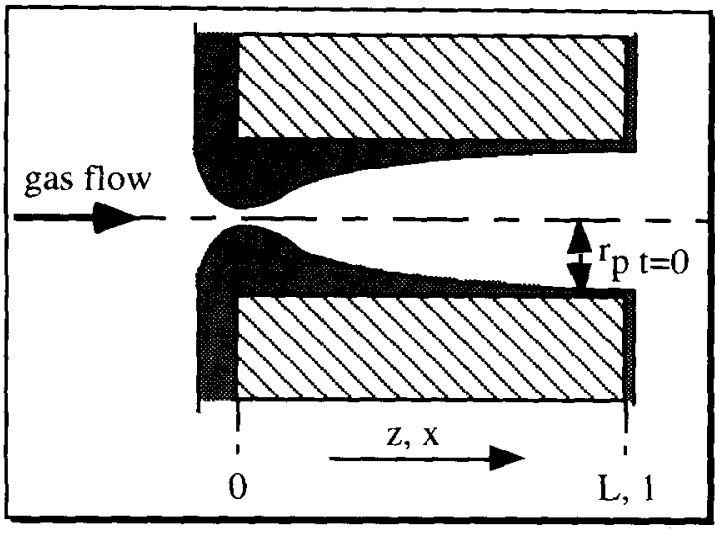

Fig. 2. A cross-section of one cylinder pore.

single raw pore volume $\left(\mathrm{m}^{3}\right), V_{\text {preform }}$ is the total raw preform volume $\left(\mathrm{m}^{3}\right)$ and $d_{\text {preform }}$ is the preform disc diameter $(\mathrm{m})$.

The preform diameter remains relatively unchanged whereas the porosity changes during infiltration. Due to deposition on the pore wall and depletion of the precursor from the gas mixture at mass transport limited conditions, the pore diameter gets larger from the gas inlet side $(z=0)$ further down the pore and thus will be dependent on $z$. This effect is visualised in Fig. 2 (Section 6).

Combination of (11) with (15) gives the velocity through one pore $u_{\mathrm{p}}\left(\mathrm{m} \cdot \mathrm{s}^{-1}\right)$ :

$$
u_{\mathrm{p}}=\frac{F t}{\epsilon A_{\text {preform }}}
$$

During the FCVI process, the driving force for the gas flow is dominated mainly by forced convection due to a pressure difference.

The FCVI operator can only adjust the total gas flow rate $F t^{\#}$ and the outlet pressure $p_{\mathrm{L}}$ to gain a certain $u_{\mathrm{p}}$ setpoint. An expression for the pore gas velocity, similar to the Hagen-Poiseuille relation, in terms of outlet pressure can be derived through combination of eqns 13 and 17:

$u_{\mathrm{p}}=\frac{d_{\mathrm{p}}^{2}}{32 \mu L} p_{\mathrm{L}}\left\{\sqrt{1+\frac{256 F t^{\#} p^{0} T \mu L}{\pi n d_{\mathrm{p}}^{4} T^{0} p_{\mathrm{L}}^{2}}}-1\right\}$

Here, the term within brackets times $p_{\mathrm{L}}$, equals the pressure drop across the pore.

\section{Effect of the Preform Microstructure on the Infiltration Behaviour}

The effect of the microstructural properties on the infiltration behaviour can be performed through correlation of these properties with the gas velocity. This latter is the prime process parameter for the description of the layer uniformity and thus density during mass transport limited growth.
In the next section, the microstructural properties are presented in detail with their effects on the infiltration behaviour of the preform.

The microstructure of the preform changes with time due to deposition within the pore network. Unless stated otherwise, the initial $(t=0)$ microstructural parameters of the raw preform are meant in all cases.

The total description of the initial preform microstructure is performed through the definition of:

- Preform permeability, indicative of the resistance of the preform for gas flow;

- Preform open porosity, the total open pore volume of the preform that is to be filled with matrix material;

- Diameter of the pores, giving the size of the openings through which the gas flows;

- Deposition area, giving the total surface area available for deposition;

- Preform thickness, the length of the path the gas molecules have to travel;

- Tortuosity, giving the complete path length in case of curved pores.

\section{Preform permeability}

The permeability $\mathrm{K}\left(\mathrm{m}^{2}\right)$ is defined as the fluid conductivity of the porous material. ${ }^{13,14}$

The permeability of a porous system is supposed to be a constant in Darcy's law.

In case the fluid is an incompressible liquid, $K$ is indeed a constant. In the case of gases, the permeability is dependent on pressure at low absolute pressure. (More details about the permeability are given in Part 2 of this study in Section 5). Because of this fact and because the liquid permeability $K_{1}$ is not a realistic useful FCVI quantity, it is necessary to express the preform microstructure in other more suitable parameters like porosity, pore size, thickness and to be more complete also in terms of tortuosity.

The permeability of the preform is, despite the facts mentioned above, still the sole parameter that gives an impression of the resistance of the structure to the gas flow.

A constraint of infiltration is that the gas permeability of the structure should be sufficiently large to be able to force the gas through it, without having the pressure at the inlet side reaching excessively high values in a short period of time.

\section{Initial preform tortuosity}

The tortuosity of a porous material $\tau(-)$, is defined ${ }^{15}$ as the square of the ratio of the average distance traversed by a particle of fluid, $L_{\text {eff }}$ to the 
direct distance through the preform, $L_{\text {preform }}$ and hence $\left(L_{\text {eff }} / L_{\text {preform }}\right) .^{2}$ The mean tortuous velocity through the bed $u_{\text {bed }}\left(\mathrm{m} . \mathrm{s}^{-1}\right)$ is:

$$
u_{\text {bed }}=\frac{u o \tau}{\epsilon}
$$

Where $u o$ is the superficial gas velocity.

It is hard to anticipate what the effect of the tortuosity will be on the density of the composite after infiltration without knowing the exact microstructure. As a first approximation it is anticipated that a preform with $\tau>1$ can be compared with a thicker preform and that effects are similar. Large tortuosity will yield low density due to larger depletion effects.

As the pores are not straight and uniform in diameter, the section with the largest resistance (small diameter or high angle) can cause early blocking of the pore resulting in early termination of the infiltration process and thus low density.

\section{The Relative Composite Density as a Function of Process and Microstructural Parameters}

During the densification process, matrix material is deposited onto the pore wall and the layer thickness of the matrix will decrease exponentially with $z$, see Fig. 2 .

Obviously, during the infiltration process, the deposition behaviour changes due to the reduction of the pore size and non-uniform deposition on the pore wall.

Modelling of the infiltration process is therefore more accurate when all conditions and pore dimensions are described both time and position dependent.

The simple equations derived in the previous sections are no longer sufficient. For position dependence, some basic differential equations were derived. This is described in Appendix B.

In summary, for a pore of length $L(\mathrm{~m})$ and radius $r(\mathrm{~m})$ at temperature $T(\mathrm{~K})$ where MTS (denoted with $S$ ) reacts at the pore wall, the three principal differential equations are:

$$
\begin{gathered}
\frac{\partial p_{x}}{\partial x}=\frac{-8 \mu L K F t^{\#}}{n \pi p_{\mathrm{x}} r_{\mathrm{x}}^{4}} \\
\frac{\partial C_{S x}}{\partial x}=-2 L C_{\mathrm{Sx}}\left[\frac{n \pi k_{s} r_{\mathrm{x}} p_{\mathrm{x}}}{F t^{\#} K}+\frac{4 \mu K F t^{\#}}{n \pi p_{\mathrm{x}}^{2} r_{\mathrm{x}}^{4}}\right] \\
\frac{\partial r_{x}}{\partial t}=\frac{-m_{\mathrm{SiC}}}{\rho_{\mathrm{SiC}}} k_{\mathrm{s}} C_{\mathrm{Sx}} \\
\text { with } K=\frac{T p^{0}}{T^{0}}(\mathrm{~Pa})
\end{gathered}
$$

and $x(-)$ is the dimensionless axial distance in the pore.

Simultaneous solution of these equations with the variables $p_{\mathrm{x}}(\mathrm{Pa}), C_{\mathrm{Sx}}\left(\right.$ mole. $\left.\mathrm{m}^{-3}\right)$ and $r_{\mathrm{x}}(\mathrm{m})$, allows the calculation of the evolution of the pore diameter as a function of time and position in axial direction. After the simulated infiltration process has ended the resulting composite microstructure can be estimated.

The open volume $V_{\mathrm{o}}$ within the preform after infiltration $\left(\mathrm{m}^{3}\right)$ is given by the general expression for a solid of revolution:

$$
V_{0}=n \pi L \int_{0}^{1} r_{\mathrm{x}}^{2} \mathrm{~d} x
$$

with $n$ the (constant) number of pores in the preform (eqn 16) and $r_{\mathrm{x}}$ represents the pore radius after infiltration.

The mass of the total amount of deposited matrix in the preform $M_{\mathrm{in}}(\mathrm{kg})$, is the matrix volume multiplied by the theoretical density of the $\mathrm{SiC}$ matrix material $\left(3 \cdot 2 \times 10^{3} \mathrm{~kg} \cdot \mathrm{m}^{-3}\right)$.

$$
M_{\text {in }}=\rho_{\mathrm{SiC}}\left(n V_{\mathrm{p}}-V_{0}\right)
$$

where $V_{\mathrm{p}}$ is the volume of one raw cylindrical pore and equals $\pi r_{p}^{2} L$.

Furthermore, the $\mathrm{SiC}$ matrix is not only deposited in the porous preform but moreover, on the front surface of the preform as well. The mass of the deposited $\mathrm{SiC}$ on its front side, neglecting the initial surface porosity, can be roughly estimated with:

$$
M_{\text {on }}=\rho_{\mathrm{SiC}} \pi r_{\text {preform }}^{2}\left(r_{\mathrm{p}}-r_{\mathrm{a}}\right)
$$

with $r_{\mathrm{a}}$ the radius of the pore mouth after infiltration $(\mathrm{m})$ and $r_{\mathrm{p}}$ the radius of the raw pore $(\mathrm{m})$. The porosity of the preform after infiltration $\epsilon_{\mathrm{c}}$ $(\mathrm{vol} \%)$ is:

$$
\epsilon_{\mathrm{c}}=\frac{V_{0}}{V_{\text {preform }}}
$$

Here, $V_{\text {preform }}$ is the volume $\left(\mathrm{m}^{3}\right)$ of the raw preform (without the coating on the front side). The relative bulk composite density is defined here as:

$$
\rho_{\text {brel }}=100-\epsilon_{\mathrm{c}}
$$

\section{The Model Set-up}

\section{General assumptions and model input}

In the simulation results described in Section 8, assumptions are made in order to gain a model that can be solved easily and is easy to use and understand. These assumptions are:

(1) Mass transport of the gaseous species takes 
place by forced convection in axial direction only. Axial dispersion and radial convection or diffusion (either ordinary or Knudsen) are not taken into account. This model is thus ID.

(2) The forced convection takes place in the viscous laminar flow regime.

(3) The gas behaves ideally.

(4) Stefan flow due to the reaction stoichiometry is neglected since dilution of the precursor compound in the gas mixture is generally large.

(5) The surface kinetics follow a first order relationship with respect to the MTS concentration. The reaction rate coefficient is assumed to be independent of total pressure.

(6) Homogeneous gas phase reactions do not occur.

(7) The initial pores are cylindrical and monosized and the tortuosity equals 1 .

The required input parameters for the modelling are:

Initial preform porosity (volume fraction), initial preform thickness $(\mathrm{m})$, diameter of the preform disc (m), activation energy of the first order deposition reaction $\left(\mathrm{J} . \mathrm{mole}^{-1}\right)$, pre-exponential factor $\left(\mathrm{m} . \mathrm{s}^{-1}\right)$, preform temperature $(\mathrm{K})$, outlet pressure $(\mathrm{Pa})$, precursor inlet molar fraction, total gas flow rate at STP $\left(\mathrm{m}^{3} \cdot \mathrm{s}^{-1}\right)$ and pressure shut off point (Pa). The latter is defined in Part 2 of this study (eqn 10).

The only input microstructural parameter that is required and initially unknown is the pore radius $r_{\mathrm{p}}$ at $t=0(\mathrm{~m})$. Through comparison of experimental infiltration durations and simulation results, this $r_{\mathrm{p}}$ can be fine-tuned to gain a matching between experiments and model.

\section{Calculation algorithm}

After input of the process conditions, the constants are calculated: $k_{\mathrm{s}}, \mu$ at the selected temperature and the number of pores $n$ (eqn 16). An initial pore diameter is selected and an initial iteration step of $5 \mathrm{~s}$ was chosen. At these conditions, the pressure within the pore as a function of position is calculated through numerical integration of eqn 21 . Hereto, the total pore is split into 40 equal length segments. Simultaneously, the MTS concentration (eqn 22) within the pore is calculated numerically and allows the estimation of the deposition rate in the pore and the local reduction in pore radius (eqn 23).

With this reduced pore size, a next time step is selected and again the pressure, concentration and growth rate are calculated for each of the 40 segments.

As the inlet pressure starts to increase more rapidly, the time step is reduced to gain higher accuracy. The whole iteration is terminated when the selected shut off pressure drop setpoint is reached. At the final pore dimensions, the relative density or porosity of the preform, the deposited mass of $\mathrm{SiC}$ in and on the preform and final pore mouth radius are calculated.

The results of the model calculations as a function of process conditions are presented in Section 8 .

\section{Modelling Results}

Due to the large amount of assumptions and simplifications made, this model can only simulate rough trends in infiltration time and composite density as a function of time and process or microstructural conditions. It is thus not intended to give quantitative information.

\section{Nominal simulation conditions}

In the following figures the results are summarised of the simulations performed at nominal process conditions using a standard preform model plate:

Temperature: $1273 \mathrm{~K}$, outlet pressure: 50 torr, total gas flow rate: $1 \mathrm{slm}$, MTS inlet mole fraction: $0 \cdot 15$, preform porosity: $50 \%$, initial preform thickness: $3 \mathrm{~mm}$, preform diameter $8 \mathrm{~cm}$, initial pore diameter: $500 \mu \mathrm{m}$, pressure shut off difference: $100 \pm 10$ torr, activation energy: $400 \mathrm{~kJ} \cdot \mathrm{mole}^{-1}$ and pre-exponential factor: $2.1 \times 10^{14} \mathrm{~m} \cdot \mathrm{s}^{-1}$ of the first order reaction. ${ }^{16}$ The dynamic viscosity of the gas mixture is estimated through: $\mu(T)=2.9 \times 10^{-7}+$ $4.8 \times 10^{-8} T-1.6 \times 10^{-11} T^{2}+3.2 \times 10^{-15} T^{3}$ in Pa.s. Initial iteration step size: $5 \mathrm{~s}$.

\section{IFCVI process as a function of time}

As infiltration proceeds and the pores become smaller, the process conditions change.

In Fig. 3, the simulated change in inlet pressure and inlet and outlet MTS concentration are presented.

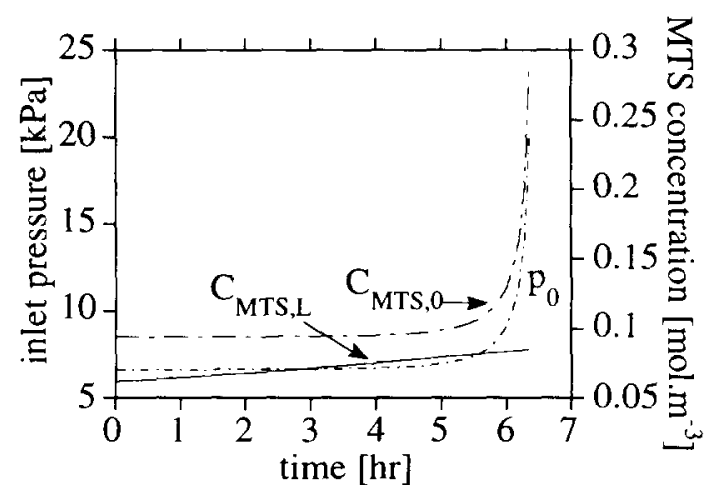

Fig. 3. Simulation result of the inlet pressure, the inlet and outlet MTS concentration (resp. $\mathrm{C}_{\mathrm{MTS} .0}$ and $\mathrm{C}_{\mathrm{MTS} . \mathrm{L}}$ ) of a model preform at nominal conditions. 


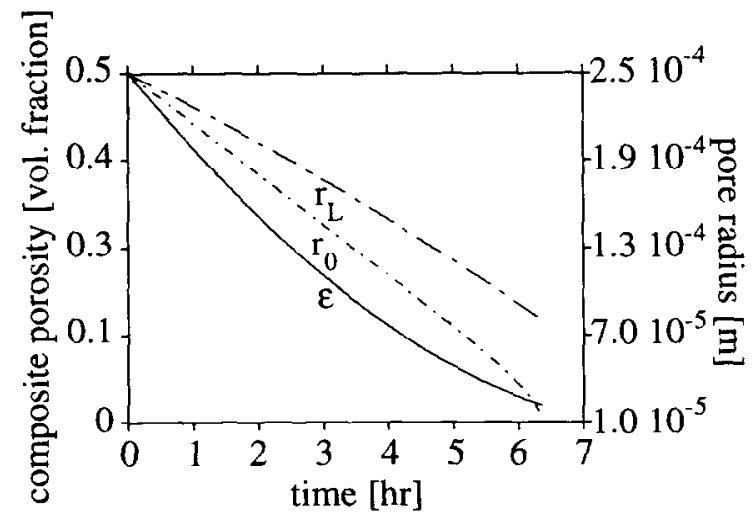

Fig. 4. Simulation result of the inlet and outlet radius (respectively $r_{0}$ and $r_{\mathrm{L}}$ and porosity of a model preform at nominal conditions.

The outlet pressure remains constant at 6.65 $\mathrm{kPa}$ ( $=50$ torr). Initially the inlet pressure hardly rises, but as soon as a critical pore size is reached, the pressure increases rapidly.

Combined with the increase of the inlet pressure, the inlet concentration and the gas velocity are increasing.

In general, more uniform deposition occurs due to the increase in gas velocity through the pores so that depletion effects are less severe in a later stage of the infiltration process. This effect is demonstrated in Fig. 3 by the increase of $\mathrm{C}_{\mathrm{MTS}, \mathrm{L}}$.

Clearly, as the pore is becoming smaller, the porosity decreases (Fig. 4). At first, decrease is rapid, but due to the reduction of the deposition (pore mantle) area, porosity is only decreasing relatively slowly later on.

\section{Total gas flow rate}

Increase in total gas flow rate and thus increase of the mass transport rate, yields a larger matrix growth rate and reduction in infiltration time (Fig. 5). Subsequently, the uniformity of the layer profile is enhanced, since the gas velocity is enlarged.

When the kinetics are becoming rate controlled, further increase of $\mathrm{Ft}^{*}$ no longer has an effect on

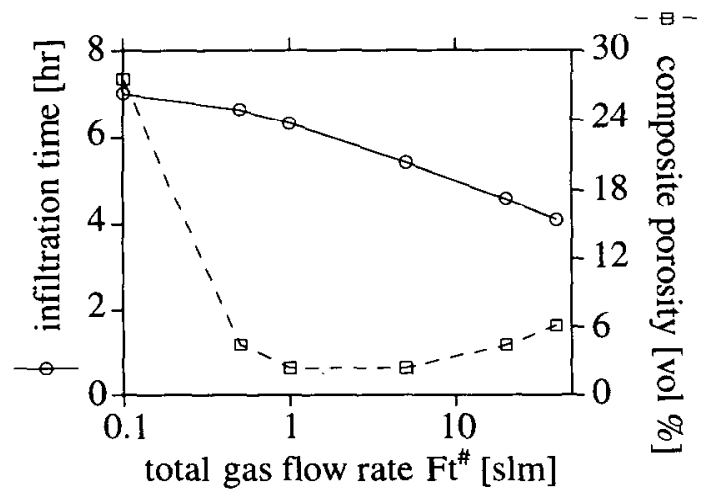

Fig. 5. The simulated infiltration time and composite porosity after infiltration of a model preform at nominal conditions versus total gas flow rate.

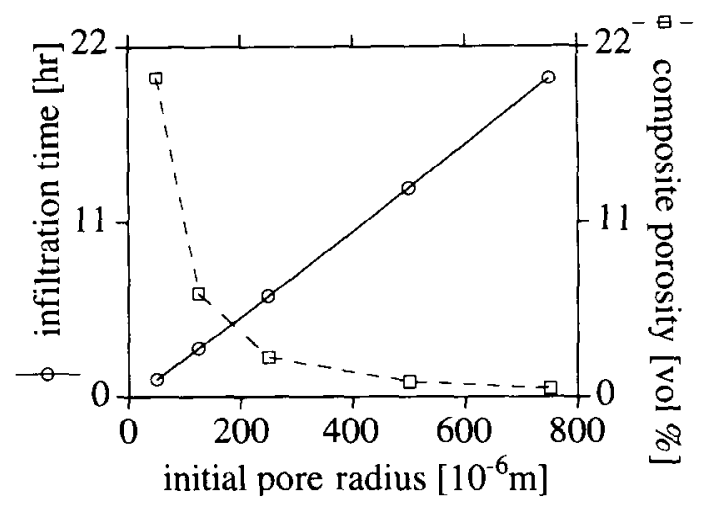

Fig. 6. The simulated infiltration time and composite porosity after infiltration of a model preform at nominal conditions versus initial pore radius.

the layer uniformity, but the increase of the initial pressure drop at large $F^{H}$ will yield a reduction in $G_{\mathrm{L}} / G_{0}$ ratio and thus increase the composite porosity. In summary, a minimum will exist in composite porosity.

\section{Initial pore size}

When $L / d_{\mathrm{p}}$ is relatively small, matrix growth is limited by the chemical reaction (eqn 8 ) and thus occurs at large $r_{\mathrm{p}}$. Increase of $r_{\mathrm{p}}$ as demonstrated in Fig. 6, linearly increases infiltration time, whereas composite porosity decreases through the increase of the gas velocity (eqn 13).

\section{Outlet pressure}

Similar to Fig. 5, in Fig. 7 an optimum (at 6.65 $\mathrm{kPa}$ outlet pressure) in composite density is found when the outlet pressure is increased from 13.3 to $1.0 \times 10^{5} \mathrm{~Pa}(0 \cdot 1-760$ torr $)$. At high outlet pressure, (i.e. $\geq 20 \mathrm{kPa}$ ), the gas velocity is low (eqn 11) so that depletion of the precursor gas is high along the pore length. Non-uniform deposition is the consequence. At low outlet pressure $\left(p_{\mathrm{L}}=0.133 \mathrm{kPa}\right)$ and thus at large gas velocity, the pressure drop value across the preform is of similar magnitude as the outlet pressure.

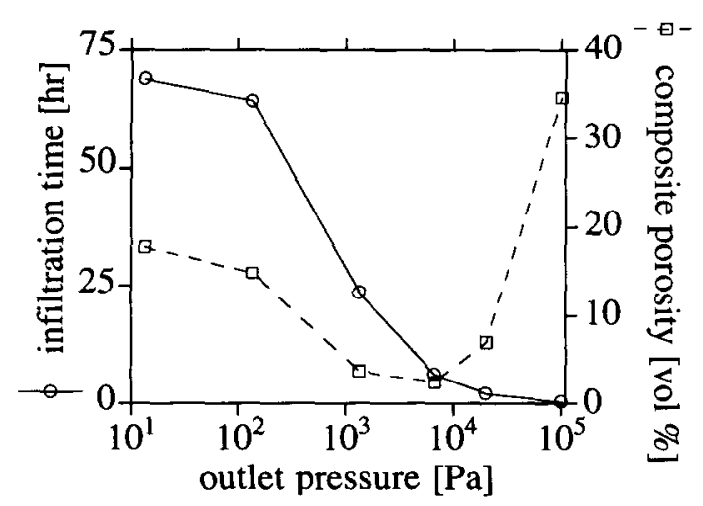

Fig. 7. The simulated infiltration time and composite porosity after infiltration of a model preform at nominal conditions versus total outlet pressure. 
The large gas velocity will yield large density, but on the other hand when $p_{0}>p_{\mathrm{L}}$ and thus $G_{0}$ $>G_{\mathrm{L}}$ uniformity will be poor. It is speculated that growth is limited by the chemical kinetics at these large gas velocities, since mass transport to the surface is very fast. Here, the matrix growth is not dependent on the gas velocity but on the local total pressure only.

Infiltration time is reduced if the total pressure and thus concentration is increased, because matrix growth is enhanced.

\section{Temperature}

At low temperature, deposition is kinetically controlled. Increase of temperature decreases infiltration time rapidly, whereas growth becomes mass transport limited infiltration time is less severely reduced (Fig. 8). As stated before, kinetically controlled growth is preferred because depletion (at low T) is low and uniformity is high.

\section{Precursor input mole fraction}

Increase of the MTS fraction in the gas mixture has no effect on the relative composite density. In relative terms, the uniformity is not affected by the absolute value of the MTS fraction within the gas mixture, neither in the mass transport limited nor in the kinetically controlled regime, as coincides with theoretical considerations.

However, infiltration time is reduced inversely proportional with the MTS fraction due to the increase of precursor concentration.

\section{Preform porosity}

The raw preform porosity is changed by alteration of the amount of pores. The gas stream is split into smaller portions, so that when the porosity is increased it will have to flow through a larger pore area. This reduction in gas velocity (eqn 11) yields a larger composite porosity in an almost linear fashion.

At low mass transport rate (high porosity) infiltration will take more time.

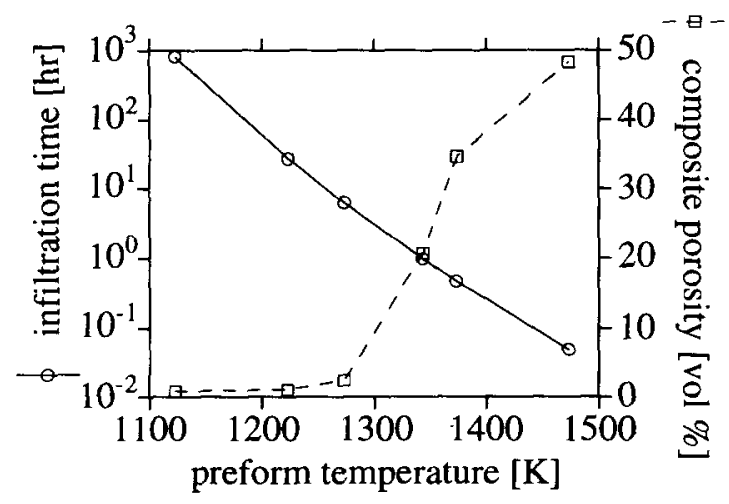

Fig. 8. The simulated infiltration time and composite porosity after infiltration of a model preform at nominal conditions versus preform temperature.

\section{Initial preform thickness}

A thicker preform disc will be infiltrated in the same time as a thin disc, as long as the deposition rate in the pore mouth is unaffected, i.e. $p_{0}$ remains unchanged.

A thick preform will, however, yield a larger initial pressure drop across the preform as compared to a thin preform at the same flow rate. Depletion of the MTS from the gas mixture is morc scvercly affecting the composite density when the pores are longer.

\section{Preform diameter}

As the preform diameter or cross flow area A gets larger, the gas velocity is decreased (eqn 11). Subsequently, composite porosity is increased. As a consequence, the infiltration time will increase since mass transport of the gas species is reduced.

\section{Discussion and Conclusions}

Despite the better and more comprehensive numerical 2D models used by others, the developed simple ID model gives a good insight in the relative complex FCVI process without using a black box approach. The simulation variables correspond to the process variables that can be adjusted by the opcrator providing a direct correlation between experiments and model.

The capability of this model is to predict trends in filtration time and composite density that might be expected when process conditions, preform structure or dimensions are varied.

The general model trends are summarised in Table 1.

The simulation results show that uniformity of the matrix deposition can be improved by allowing

Table 1. The infiltration behaviour parameter dependence on initial process conditions and raw preform structure. The matrix growth is either limited by the surface kinetics or the mass transport rate.

\begin{tabular}{lccc} 
& & \multicolumn{2}{c}{ Composite density } \\
\cline { 3 - 4 } $\begin{array}{l}\text { Parameter } \\
\text { increase }\end{array}$ & $\begin{array}{c}\text { Infiltration } \\
\text { time }\end{array}$ & $\begin{array}{c}\text { Kin. } \\
\text { contr. }\end{array}$ & $\begin{array}{c}\text { Conv. } \\
\text { contr. }\end{array}$ \\
\hline$u_{\mathrm{p}}$ & $\downarrow$ & $\downarrow$ & $\uparrow$ \\
$F t^{\#}$ & $\downarrow$ & $\downarrow$ & $\uparrow$ \\
$p_{\mathrm{L}}$ & $\downarrow$ & $\uparrow$ & $\downarrow$ \\
$T$ & $\downarrow$ & - & $\downarrow$ \\
$X_{\mathrm{MTS}}$ & $\downarrow$ & - & - \\
$d_{\mathrm{p}}$ & $\uparrow$ & - & $\downarrow$ \\
$d_{\text {preform }}$ & $\downarrow$ & - & $\downarrow$ \\
$\epsilon$ & $\uparrow$ & - & $\downarrow$ \\
$L$ & $\downarrow$ & &
\end{tabular}

*Assuming a first order reaction. 
a large pore gas velocity in combination with a low inlet to outlet pressure ratio.

The first Damköhler number should be small. This implies operation where the gas velocity is so fast that matrix growth is just within the kinetically controlled growth regime. This generally occurs at: medium total gas flow rate, medium outlet pressure, low temperature and arbitrary precursor content in the gas mixture (with the constraint however that pure $\mathrm{SiC}$ will be formed, so the ratio $\mathrm{H}_{2}$ /MTS must not be too low).

The preform microstructure that is best suited for FCVI processing to gain a large composite density has a low open porosity, is thin and contains pores of large diameter. In general the gas permeability of the porous structure should be sufficiently large to enable the gas to flow through the structure easily. The best starting preform microstructure (without considering mechanical aspects) implies: low porosity, thin walled structures, large pore diameter, straight continuous pores and sufficient permeability.

For economical reasons, the infiltration time required to reach a certain homogeneous density should be kept as low as possible. Obviously, a high matrix growth rate across the entire pore length is essential. Increase of the matrix growth rate can be tailored by increase of the precursor mole fraction in the gas mixture without affecting the uniformity of the deposition profile. Increase of the temperature will also enhance densification rates, but will negatively affect the uniformity of the matrix distribution. Rapid infiltration is also enhanced through increase of the gas velocity. Lowering the total pressure will negatively affect the matrix growth rate and thus increase of the total gas flow rate is more suitable for this purpose.

In summary, the global optimum process settings for both rapid and dense composites are thus:

- high total gas flow rate;

- medium outlet pressure;

- medium temperature;

- high precursor molar fraction;

the best starting preform microstructure implies:

- low porosity;

- thin walled structures;

- straight continuous pores;

- medium pore size;

- sufficient permeability.

Unfortunately, it can be concluded that rapid densification does not coincide with high composite homogeneity and density.

For industrial application of the FCVI process, the real processing optimum depends on the specification of the desired composite material, especially its density. A high density can be achieved, but the time consumption will be large. This extra energy and material consumption should thus be compared with the extract value of the material for the specific application.

\section{References}

1. Janssen, L. P. B. M. \& Warmoeskerken, M. M. C. G., Transport Phenomena Data Companion, Delftse Uitgevers Maatschappij, Delft, (1991).

2. Levenspiel, O., Chemical Reaction Engineering, Wiley, New York, (1972).

3. v.d. Brekel, C. H. J., Fonville, R. M. M., van der Straten, P. J. M. \& Verspui, G., Proc. 8th Int. Conf. on $C V D$, Electrochem Soc., (1981).

4. Rossignol, J. Y., Langlais, F. \& Naslain, R., Proc. 9th Int. Conf. on CVD, ed. Mc. D. Robinson, The Electrochem. Soc. (1984), p. 596-614.

5. Roman, Y. G., de Croon, M. H. J. K. \& Metselaar, R., submitted to the J. Eur. Ceram. Soc.

6. Chung, G. Y. \& McCoy, B. J., J. Am. Ceram. Soc., 74 (1991) 746-51.

7. Tai, N. \& Chou, T., J. Am. Ceram. Soc, 72 (1989) 414-20.

8. Fedou, R., Langlais, F. \& Naslain, R., J. Mat. Synthesis and Processing, 1 (1993) 43-52. ibid Ref. 2. 61-74.

9. Lin, Y. S. \& Burggraaf, A. J., Chem. Eng. Sci., 46 (1991) 3067-80.

10. Gupte, S. M. \& Tsamopoulous, J. A., J. Electrochem. Soc., 137 (1990) 1626-38

11. Starr, T., Ceram. Sic. Eng., 9 (1988) 803-12.

12. Starr, T., Proc. 10th Int. Conf on CVD, ed. G. W. Collen, The Electrochem. Soc. (1987), p. 1147-55.

13. Bear, J., Dynamics of fluid in porous media, Elsevier, New York, (1972)

14. Collins, R. E., Flow of fluids through porous materials, Reinhold Publ. Corp., (1961).

15. Carman, P. C., Trans. Inst. Chem. Eng. London, 15 (1937) 150.

16. Roman, Y. G., Forced flow chemical vapour Infiltration, $\mathrm{PhD}$ thesis Eindhoven University of Technology, Eindhoven, The Netherlands, (1994).

\section{Appendix A. Time Dependent Precursor Concentration in a Cylindrical Model Pore}

The cylindrical model pore can be assumed to be a steady state plug flow reactor in which forced convection is present and chemical reaction takes place on the pore wall. Stefan flow is neglected since the dilution of the $\mathrm{SiCH}_{3} \mathrm{Cl}_{3}$ in the carrier gas (hydrogen) is high. If it is assumed that the pore gas velocity is constant throughout the pore and independent of axial position, the following simplified analytical solution of the concentration of MTS within the pore can be derived.

The mass balance for a differential element of the pore with volume $d V$ :

$$
F_{\mathrm{Sz}}=\left(F_{\mathrm{S}}+\mathrm{d} F_{\mathrm{S}}\right)_{z}+\left(-r_{\mathrm{S}} \mathrm{d} V\right)
$$

where ' $\mathrm{S}$ ' denotes $\mathrm{SiCH}_{3} \mathrm{Cl}_{3}, F_{\mathrm{Sz}}$ the molar flow rate $\mathrm{S}$ (moles. $\mathrm{s}^{-1}$ ) and equals $C_{\mathrm{Sz}} u_{\mathrm{z}} A_{z}$ since $\mathrm{S}$ is heavily diluted in the carrier gas. $C_{\mathrm{Sz}}, u_{\mathrm{z}}$ and $A_{\mathrm{z}}$ are respectively the concentration of $\mathrm{S}\left(\right.$ moles. $\mathrm{m}^{-3}$ ), 
the gas velocity $\left(\mathrm{m} . \mathrm{s}^{-1}\right)$ and the cross flow area $\left(\mathrm{m}^{2}\right)$ at a location in axial direction $z$ within the pore. Also:

$$
C_{\mathrm{Sz}}=\frac{F_{\mathrm{S} 0}\left(1-Y_{\mathrm{Sz}}\right)}{u_{\mathrm{z}} A_{\mathrm{z}}}
$$

where $Y_{\mathrm{S}}$ is the conversion of $\mathrm{S}$.

The reaction rate $\left(\right.$ moles. $\mathrm{m}^{-3} \cdot \mathrm{s}^{-1}$ ) of $\mathrm{S}$ on the pore wall is:

$$
-r_{\mathrm{S}}=k_{\mathrm{v}} C_{\mathrm{Sz}}=\frac{k_{\mathrm{v}} F_{\mathrm{S} 0}\left(1-Y_{\mathrm{Sz}}\right)}{u_{\mathrm{z}} A_{\mathrm{z}}}
$$

where $k_{\mathrm{v}}$ is the volumetric reaction rate coefficient for the first order reaction $\left(\mathrm{s}^{-1}\right)$.

Rewriting A1 yields:

$$
-\mathrm{d} F_{\mathrm{S} z}=F_{\mathrm{S} 0} \mathrm{~d} Y_{\mathrm{Sz}}=-r_{\mathrm{S}} \mathrm{d} V
$$

and combination with $\mathrm{A} 3$ gives:

$$
\mathrm{d} Y_{\mathrm{S} z}=\frac{-r_{\mathrm{S}} \mathrm{d} V}{F_{\mathrm{S} 0}}=\frac{k_{v}\left(1-Y_{\mathrm{S} z}\right) \mathrm{d} V}{u_{\mathrm{z}} A_{\mathrm{z}}}
$$

After grouping of the terms and with $\mathrm{d} V=A \mathrm{~d} z$ for a straight pore:

$$
\int_{0}^{Y_{\mathrm{S}_{2}}} \frac{1}{1-Y_{\mathrm{S} z}} \mathrm{~d} Y_{\mathrm{Sz}}=\int_{0}^{Z} \frac{k_{\mathrm{v}}}{u_{t}} \mathrm{~d} z
$$

Here, the reaction rate coefficient $k_{\mathrm{v}}$ is based on the volume of the pore. In terms of surface area of the pore $k_{\mathrm{s}}\left(\mathrm{m} . \mathrm{s}^{-1}\right)$ is calculated through: $k_{\mathrm{v}} V=k_{\mathrm{s}}$ $A$ with $A$ representing the deposition or mantle area of the straight pore $\left(\mathrm{m}^{2}\right) . k_{\mathrm{v}}$ is thus $4 k_{\mathrm{s}} / d_{\mathrm{p}}$, where $d_{\mathrm{p}}$ is the pore diameter of the straight pore (m).

Equation A6 can be integrated from pore mouth to length $z$ and combined with (eqn A2):

$$
1-Y_{\mathrm{Sz}}=\frac{C_{\mathrm{Sz}} u_{\mathrm{z}} A_{\mathrm{z}}}{C_{\mathrm{S} 0} u_{0} A_{0}}
$$

to yield:

$$
\frac{C_{\mathrm{sz}} u_{\mathrm{z}} A_{\mathrm{z}}}{C_{\mathrm{s} 0} u_{0} A_{0}}=\exp \left(-4 k_{\mathrm{s}} \int_{0}^{z} \frac{1}{u_{\mathrm{z}} d_{\mathrm{pz}}} \mathrm{dz}\right)
$$

This equation reduces to a simpler one when the pressure across the pore axis is assumed to be linearly decreasing from $p_{0}$ towards $p_{\mathrm{L}}$ and the pore is straight so that $u_{z}$ and $\mathrm{d}_{\mathrm{pz}}$ are independent of $z$. At constant pressure and for an incompressible gas, the continuity equation is $\rho_{z} u_{z} A_{z}=\rho_{0} u_{0} A_{0}$. A very rough estimation of the concentration profile is:

$$
\frac{C_{\mathrm{Sz}}}{C_{\mathrm{S} 0}}=\exp \left(\frac{-4 k_{\mathrm{s}} z}{d_{\mathrm{p}} u_{\mathrm{p}}}\right)
$$

where $u_{\mathrm{p}}$ and $d_{\mathrm{p}}$ are based upon the entrance pore diameter conditions $(z=0)$.
Appendix B. Time and Position Dependent Precursor Concentration in a Cylindrical Model Pore

It may be clear that due to non-uniform deposition on the pore wall (as a consequence of depletion and the pressure gradient), the total gas flow rate, total pressure, MTS concentration and pore radius are all a function of position along the pore axis $z$.

The next section presents the derivations of three differential equations and are: the total gas flow rate, total pressure within the pore, the MTS concentration and the pore radius as a function of position in axial direction. These equations are solved simultaneously using a Mathematica $^{\dagger}$ numerical routine based upon the Runge Kutta method.

Recall the inflow gas velocity (eqn 11):

$$
u_{\mathrm{p} z}=\frac{F t^{\#} T p^{0}}{n A T^{0} p_{\mathrm{z}}}=\frac{F t^{\#} T p^{0}}{n \pi r_{\mathrm{z}}^{2} T^{0} p_{\mathrm{z}}}
$$

and the Poiseuille flow eqn 13 as:

$$
u_{\mathrm{pz}}=\frac{-r_{z}^{2}}{8 \mu} \frac{\partial p_{\mathrm{z}}}{\partial z}
$$

Rewriting the equations in terms of dimensionless axial distance $x: x=z / L>\mathrm{d} z=L \mathrm{~d} x$, equation $\mathrm{B} 2$ becomes:

$$
u_{\mathrm{pz}}=\frac{-r_{x}^{2}}{8 \mu L} \frac{\partial p_{\mathrm{x}}}{\partial x}
$$

A combination of (B1) and (B3) yields the position dependent pressure differential equation:

$$
\frac{\partial p_{\mathrm{x}}}{\partial x}=\frac{-8 \mu L K F t^{\#}}{n \pi p_{x} r_{\mathrm{x}}^{4}} \text { with } K=\frac{T p^{0}}{T^{0}}
$$

The mass balance for a differential element of the pore with volume $\mathrm{d} V$ :

$$
F_{\mathrm{Sz}}=\left(F_{\mathrm{S}}+\partial F_{\mathrm{S}}\right)_{\mathrm{z}}+\left(-r_{\mathrm{S}} \partial V\right)
$$

where $\mathrm{SiCH}_{3} \mathrm{Cl}_{3}$ is denoted as ' $\mathrm{S}$ ' and $F_{\mathrm{Sz}}$ is the molar flow rate of $\mathrm{S}$ (moles. $\mathrm{s}^{-1}$ ).

The first order reaction rate of disappearance of $\mathrm{S}, r_{\mathrm{S}}\left(\right.$ moles. $\left.\mathrm{m}^{-3} \cdot \mathrm{s}^{-1}\right)$ on the pore wall is:

$$
r_{\mathrm{S}}=-k_{\mathrm{v}} C_{\mathrm{S} z}
$$

Here, the reaction rate cocfficient $k_{\mathrm{v}}$ is bascd on the volume of the pore. In terms of surface area of the pore, $k_{\mathrm{s}}\left(\mathrm{m} \cdot \mathrm{s}^{-1}\right)$ is calculated through: $k_{\mathrm{v}} V=k_{\mathrm{s}}$ $A$, where $A$ represents the deposition (or pore mantle) area $\left(\mathrm{m}^{2}\right)$ and thus $k_{\mathrm{v}}{ }^{\prime}=\frac{2 \mathrm{k}_{\mathrm{s}}}{r_{\mathrm{z}}}$. Rewriting $\mathrm{B} 5$ yields:

$$
\partial F_{\mathrm{Sz}}=r_{\mathrm{S}} A_{\mathrm{z}} \partial \mathrm{z}=r_{\mathrm{S}} \pi r_{\mathrm{z}}^{2} \partial \mathrm{z}
$$

${ }^{\dagger}$ Mathematica is software of Wolfram Research Inc. 


$$
\begin{gathered}
\partial F_{\mathrm{Sz}}=-k_{\mathrm{S}} C_{\mathrm{Sz}} 2 \pi r_{\mathrm{z}} \partial \mathrm{z} \text { with } \partial \mathrm{z}=L \partial x \\
\frac{\partial F_{\mathrm{Sx}}}{\partial x}=-2 \pi k_{\mathrm{s}} L C_{\mathrm{S} \mathrm{x}} r_{\mathrm{x}}
\end{gathered}
$$

$F_{\mathrm{Sx}}$ equals $\left(C_{\mathrm{Sx}} u_{\mathrm{x}} A_{\mathrm{x}}\right)$ if the dilution of $\mathrm{S}$ in the carrier gas is high. $C_{\mathrm{Sx}}, u_{\mathrm{x}}$ and $A_{\mathrm{x}}$ are respectively the concentration of $\mathrm{S}$ (moles. $\mathrm{m}^{-3}$ ), the gas $\left(\mathrm{m} . \mathrm{s}^{-1}\right)$ velocity and the cross flow area $\left(\mathrm{m}^{2}\right)$ at a location in axial direction within the pore. It is known that:

$$
C_{\mathrm{Sx}}=\frac{n F_{S x}}{F t_{\mathrm{x}}}=\frac{n F_{S x} p_{x}}{F t^{\#} K}
$$

where $F_{\mathrm{Sx}}$ is the molar and $F t_{\mathrm{x}}$ is the volumetric flow rate and equals $\left(u_{\mathrm{x}} A_{\mathrm{x}}\right)$ and $p_{\mathrm{x}}$ is the total pressure in the pore as a function of $x$. Differentiation on both sides yields:

$$
\frac{\partial F_{S x}}{\partial x}=\frac{F t^{\#} K}{n} \frac{\partial \frac{C_{\mathrm{Sx}}}{p_{x}}}{\partial x}
$$

and with the chain rule:

$$
\frac{\partial F_{S x}}{\partial x}=\frac{F t^{\#} K}{n}\left[\frac{1}{p_{\mathrm{x}}} \frac{\partial C_{\mathrm{Sx}}}{\partial x}-\frac{C_{\mathrm{Sx}}}{p_{\mathrm{x}}^{2}} \frac{\partial p_{\mathrm{x}}}{\partial x}\right]
$$

Combination of equations (B9) and (B12) yields:

$$
\frac{F t^{\#} K}{n}\left[\frac{1}{p_{\mathrm{x}}} \frac{\partial C_{\mathrm{Sx}}}{\partial x}-\frac{C_{\mathrm{Sx}}}{p_{\mathrm{x}}^{2}} \frac{\partial p_{\mathrm{x}}}{\partial x}\right]=-2 \pi k_{\mathrm{s}} L r_{\mathrm{x}} C_{\mathrm{Sx}}(\mathrm{B} 13)
$$

hence:

$$
\frac{1}{p_{\mathrm{x}}} \frac{\partial C_{\mathrm{Sx}}}{\partial x}=\frac{-2 n \pi k_{\mathrm{s}} L r_{\mathrm{x}} C_{\mathrm{Sx}}}{F t^{\#} K}+\frac{C_{\mathrm{S}_{\mathrm{x}}}}{p_{\mathrm{x}}^{2}} \frac{\partial p_{\mathrm{x}}}{\partial x}
$$

combination with equation (B4):

$$
\frac{\partial C_{\mathrm{Sx}}}{\partial x}=-2 L C_{\mathrm{Sx}}\left[\frac{\pi n k_{\mathrm{s}} r_{\mathrm{x}} p_{\mathrm{x}}}{F t^{\#} K}, \frac{4 \mu K F t^{\#}}{n \pi p_{\mathrm{x}}^{2} r_{\mathrm{x}}^{4}}\right]
$$

with the boundary condition that at $x=0$ :

$$
C_{\mathrm{S} 0}=\frac{p_{\mathrm{S} 0}}{\operatorname{Rg} T}=\frac{S_{\mathrm{S} 0 \mathrm{p} 0}}{\operatorname{Rg} T}
$$

For the change in pore radius due to deposition on its wall:

$$
\frac{\partial r_{\mathrm{x}}}{\partial t}=\frac{-m_{\mathrm{Sic}}}{\rho_{\mathrm{Sic}}} k_{\mathrm{s}} C_{\mathrm{Sx}}
$$

(B4), (B15) and (B17) and the three principal equations that depend on the three variables $p_{x}$, $C_{\mathrm{x}}, r_{\mathrm{x}}$ and can be solved simultaneously as a function of both position $x$ and time $t$ to yield the development of the matrix deposition within a cylindrical pore. 